Conclusion: The majority of ovarian cancer patients experienced symptoms of CRCI, highlighted by memory and attention problems. Sociocultural and life-and-work adjustments to cope with cancer play an important role in the CRCI symptom experience.

Poster (M21)

Vulvar and Vaginal Cancer

https://doi.org/10.3802/jgo.2021.32.S1.M21

\section{Good outcome vulvar adenocarcinoma- mucinous with pseudomyxoma type stage IVB-grade I after radical vulvectomy, bilateral inguinal lymphadenectomy, left pelvic lymphadenectomy, vulvar reconstruction (keystone flap), colostomy transversum adjuvant radiation}

\section{Sutrisno Sutrisno, Tricia Dewi Anggraeni}

Gynaecology Oncology Division, Dr. Cipto Mangunkusumo Hospital, Jakarta, Indonesia (anggi73@gmail.com)

Objective: To discuss about the role of radical vulvectomy, bilateral inguinal lymphadenectomy, left pelvic lymphadenectomy (external iliac and obturator), vulvar reconstruction (keystone flap), colostomy transversum and adjuvant radiation in vulvar adenocarcinoma of stage IVB - mucinous with pseudomyxoma type: a rare case.

Methods: The 52-year-old woman, para 3, was referred from Adyaksa Hospital with vulvar cancer. Pain in the genital area heavily increased since September 2019. Firstly, at 2017, she complained of mass in her vagina, which was $2 \mathrm{~cm}$ and initially was not painful, but it became bigger in the last 2 months, sized about $8 \mathrm{~cm}$ and felt painful. She also experienced weight loss of 8 $\mathrm{kg}$ in 2 months. She had no complaints on urination or defecation. She consumed pain killer and antibiotics to relieve her pain. In RSUPN Dr. Cipto Mangunkusumo (RSCM), systemic examination was normal, but gynecological examination showed a solid, fixed mass with rough surface covered by skin on the left labia majora $8 \times 8 \times 7 \mathrm{~cm}$, upto $1 / 3$ posterior wall of distal vagina, reddish, with indistinct boundary, easy bleeding, soft, sized $3 \times 4 \mathrm{~cm}$ extended and associated with mass in the vulva. Distance to urethra was $2 \mathrm{~cm}$, distance to anus $1.5 \mathrm{~cm}$. The RSCM histopathology was reported on October 10th, 2019, and it was histologically suitable with well differentiated adenocarcinoma intestinal type of the vulva. Magnetic resonance imaging (MRI) of whole abdomen with contrast RSCM was taken on October 17th, 2019:

- In the vulva region, a solid mass appears to sting contrast with the diffusion restriction measuring $8.7 \times 4.1 \times 7.4 \mathrm{~cm}$. The mass involves the perienum and $1 / 3$ of the distal vagina. OUE, urethra and rectum involvement was not seen. Enlargement of multiple lymph nodes in the bilateral inguinofemoral region (largest diameter on the left side $4.3 \mathrm{~cm}$ ) to bilateral external parailiacs (largest diameter on the left side $1.2 \mathrm{~cm}$ ). Lesions in the inguinofemoral region only involve subcutaneous smears, no visible cuticle defects in the region

- In the left adnexal cystic lesion was seen with a bleeding component of $5.7 \mathrm{~cm}$ in diameter. In the right adnexa there is a cystic lesion of $2 \mathrm{~cm}$ in diameter.

- Uterus anteflexed normal shape and size, does not show mass.

- The liver, gallbladder, spleen, pancreas, kidneys were normal no visible mass or focal lesions.

- Aorta was normal, there is no apparent lymphadenopathy in the paraaorta.On October 24th, 2019, The radical vulvectomy, bilateral inguinal lymphadenectomy, left pelvic lymphadenectomy (external iliac and obturator), vulvar reconstruction (keystone flap), colostomy transversum was done. On February 2020, the adjuvant radiation (28s courses of external beam radiotherapy) was done. At early 2021, colostomy was closed and repair.

Results: On August 5th, 2020, whole abdomen MRI with contrast RSCM was done, revealing no evidence of disease (complete response). At 3-6 months routine evaluation, until September 2021, there was no evidence of disease (complete response). Conclusion: The radical vulvectomy, bilateral inguinal lymphadenectomy, left pelvic lymphadenectomy (external iliac and obturator), vulvar reconstruction (keystone flap), colostomy transversum and adjuvant radiation was the best treatment option for vulvar adenocarcinoma stage IVB - mucinous with pseudomyxoma.

Poster (M22)

Miscellaneous

https://doi.org/10.3802/jgo.2021.32.S1.M22

\section{DNA content in ovarian scaffold using various tissue decellularization techniques: a model for fertility preservation in cancer patients}

Paweena Thuwanut, ${ }^{1, *}$ Christiani A. Amorim, ${ }^{2}$ Wisan Sereepapong, ${ }^{1}$ Pinyada Panyavaranant, ${ }^{1}$ Porntip Sirayapiwat ${ }^{1}$ 'Department of Obstetrics and Gynecology, Faculty of Medicine, Chulalongkorn University, Bangkok, Thailand (Paweena.T@chula.ac.th) ${ }^{2}$ Department of Gynecology, Université Catholique de Louvain, Brussels, Belgium

Objective: The present study principally aimed to compare efficacy of ionic and non-ionic detergents to eliminate DNA content in ovarian scaffold using tissue decellularization technique.

Methods: Ovarian tissues were retrieved from four premenopausal patients, age 42 to 45 years old, with gynaecologic pathological 
conditions. Use of human biological materials was approved by Institutional Review Board. Cortical and medulla tissues (average size; $0.5 \times 0.5 \times 0.2 \mathrm{~mm}$; width $\times$ length $\times$ thickness) from each patient were randomly allocated into 5 groups 1$)$ fresh control; 2) tissue decellularization using ionic detergent ( $0.5 \%$ sodium dodecyl sulfate [SDS]); 3) tissue decellularization using 1.0\% SDS; 4) tissue decellularization using non-ionic detergent $(1.0 \%$ Triton-X), and 5) tissue decellularization using 5.0\% Triton-X. After 24-hour decellularization process under various detergents, all tissues were treated with DNase 1 enzyme overnight and followed by detergent residual removal for another 24 hours. DNA concentration and detergent residuals from each tissue were evaluated using spectrophotometer (optical density 260/280 and $500 \mathrm{~nm}$, respectively).

Results: The major outcomes revealed that the lowest DNA concentration in cortical tissues was observed in $0.5 \%$ SDS group (group 2 vs. control, $229 \pm 101$ vs. $1,371 \pm 389 \mathrm{ng} / \mu \mathrm{L}$ ). In contrast to medulla, DNA content was effectively eliminated by $5.0 \%$ Triton-X (group 4 vs. control, $344 \pm 143$ vs. 1,820 \pm 431 ng/ $\mu \mathrm{L}$ ). However, detergent residuals were present in all treated tissues.

Conclusion: Ovarian scaffold might possibly be constructed either by ionic or non-ionic detergents which was depended on ovarian cell type. This technology could be further applied for in vitro follicle growth in young cancer patients who prefer to preserve their fertility.

Poster (M23)

Surgical Techniques \& Perioperative Management

https://doi.org/10.3802/jgo.2021.32.S1.M23

\section{Fluorescence ureter navigation during laparoscopic hysterectomy}

\section{Pinyada Panyavaranant, "Trainee Manchana}

Gynecologic Oncology Unit, Obstetrics \& Gynecology Department, Faculty of Medicine, King Chulalongkorn Memorial Hospital, Bangkok, Thailand (pinnpanya@gmail.com)

Recently, laparoscopic surgery has been widely employed for gynecological surgery including total laparoscopic hysterectomy. However, laparoscopic surgery may injure the ureters slightly increase urological tract injury thus leading to long-term medical complications such as fistula formation and renal failure, resulting in the need for additional treatments and increase morbidity. Nonetheless, such complications can be prevented by knowing direct visualization the location of ureters throughout the whole surgery session and carefully not to injure the ureters whether during the cutting, coagulation, or ligation. Identification of the ureters can be done by insertion of fluorescence ureteric catheters through cystoscopy prior to the surgery. Afterwards, using a laparoscope that is capable of absorbing the near-infrared spectral range together with the fluorescence imaging system, the inserted ureteric catheters are then illuminated. This procedure enables the surgeons to see the ureters clearly and thus facilitates safe surgery.

Poster (M24)

Surgical Techniques \& Perioperative Management

https://doi.org/10.3802/jgo.2021.32.S1.M24

\section{Inguinofemoral lymph node dissection for vulvar cancer}

\section{Mikio Mikami}

Department of Obstetrics and Gynecology, Tokai University School of Medicine, Isehara, Japan (mmikami@is.icc.u-tokai.ac.jp)

The skills and techniques of the surgery for vulvar cancer are derived from the traditional surgery. Especially, the inguinal lymph node dissection is an essential part of the surgery but is not common as a daily practice. It is important for a surgeon to be well acquainted before surgery with the anatomy of the femoral triangle and the skills of the dissection before surgery. I will show you the standard procedure of Inguinofemoral lymph node dissection. Before dissecting the femoral area, surgeon must identify the position of femoral artery by touching and feeling the pulse and imaging the anatomical structures beneath the fascia lata.

Poster (M25)

Gynecologic Pathology, Genetics and Epidemiology https://doi.org/10.3802/jgo.2021.32.S1.M25

\section{Genomic landscape and its correlations with immunotherapy-related biomarkers in Chinese gynecologic cancer patients}

\author{
Guoliang Li, Chaoran Xia, Shoutai Ding, Pushan Shi, \\ Zhixiang Yan, Peng Luo, Jiajia Xu* \\ Topgen Biological Medicine Technology Co., Ltd., Shanghai, China \\ (xujiajia@topgen.com.cn)
}

Objective: To explore mutational characterization, tumor mutational burden (TMB) and its correlations with gene alterations, TMB in Chinese gynecologic cancer.

Methods: In 207 samples (formalin-fixed, paraffin-embedded tissue samples) including 141 ovarian cancer (OC), 28 cervical cancer (CC) and 38 endometrial carcinoma (EC), were detected by OncoDrug-Seq 603-gene panel assay through next generation sequencing (NGS) using Illumina NovaSeq 6000.

Results: In 207 samples, the median TMB was 4.7/Mb with the range from $0 / \mathrm{Mb}$ to $248 / \mathrm{Mb}$. The median TMB of OC, CC, 